\title{
ABNORMALITIES IN NOETHERIAN RINGS
}

\author{
J. T. ARNOLD AND M. B. BOISEN, JR. ${ }^{1}$
}

\begin{abstract}
If $P \subseteq Q$ are prime ideals in some ring $R$ and if rank $Q=$ $\operatorname{rank}(Q / P)+\operatorname{rank} P+k$, then $P \subset Q$ is said to be $k$-abnormal and $k$ is called the degree of abnormality. The paper consists of two examples. The first example is a Noetherian integral domain in which the set of degrees of abnormality is unbounded. Let $P$ be a prime ideal of $R$ and set $W=\{Q \mid Q$ is a prime ideal and $P \subset Q$ is abnormal $\}$. The second example is a local domain such that $\{k \mid P \subset Q$ is $k$-abnormal for some $Q \in W\} \neq\{k \mid P \subset Q$ is $k$-abnormal for some $Q$ minimal in $W\}$.
\end{abstract}

Throughout the paper all rings considered are assumed to be commutative with unity. Let $P \subset Q$ be prime ideals in a Noetherian ring $R$. In studying the various chain conditions and related questions on $R$, it is frequently of interest to know whether it is the case that $\operatorname{rank} Q=\operatorname{rank}(Q / P)+\operatorname{rank} P$. (Cf. Corollary 2.4, Corollary 2.13, and Remark 2.33(i) of [5].) It is well known that this equality need not hold in a Noetherian ring [4, Example 2, p. 203] and it thus becomes natural to consider abnormalities in a Noetherian ring. If rank $Q=\operatorname{rank}(Q / P)+\operatorname{rank} P+k$, then $P \subset Q$ is said to be $k$-abnormal and $k$ is called the degree of abnormality. This notion is introduced and studied by E. G. Houston and S. McAdam in [2]. In [2, Theorem 1.6], Houston and McAdam prove for a given prime ideal $P$ that $\{k \mid$ there is a prime ideal $Q$ with $P \subset Q k$-abnormal $\}$ is a finite set. They then ask whether the set of all degrees of abnormalities that can occur in a Noetherian ring is finite or, equivalently, whether there is a Noetherian ring in which the set of degrees of abnormalities is unbounded [2, p. 67]. In Example 1 we construct such a ring. For a given prime ideal $P$, Houston and McAdam also show in [2, Corollary 1.9] that the set $\{Q \mid Q$ is a prime ideal such that $P \subset Q$ is abnormal $\}$ has only finitely many minimal elements. They ask [2, pp. 67-68] whether, in a Noetherian ring, every degree of abnormality for any $P \subset Q$ occurs for some choice of $Q$ among these minimal elements. Example 2 illustrates that this need not be the case even for local domains.

The examples presented in this paper rely heavily on Nagata's Example 2 [4, pp. 203-205]. The following proposition is essential to the execution of the construction.

Received by the editors May 8, 1978.

AMS (MOS) subject classifications (1970). Primary 13E05, 13C15.

Key words and phrases. Prime ideal, Noetherian ring, local ring, rank.

'We are grateful to the referee for several helpful comments.

$0002-9939 / 79 / 0000-0001 / \$ 02.50$

(c) 1979 American Mathematical Society 
Proposition. Let $\left(T, M_{1}, \ldots, M_{n}\right)$ be a semilocal domain containing a field $K$ such that $T / M_{i}$ is finite over $K$ for all $i$. Then $R=K+M$, where $M=\cap{ }_{i=1}^{n} M_{i}$, is a local domain with maximal ideal $M$. The contraction map from the ideals of $T$ to those of $R$ induces a homeomorphism from $\operatorname{spec}(T) \backslash$ $\left\{M_{1}, \ldots, M_{n}\right\}$ onto spec $R \backslash\{M\}$. Furthermore, $T$ is integral over $R$.

This proposition is essentially the same as (E2.1) from Nagata [4, p. 204] except for our statement relating the prime spectrums of $R$ and $S$ which is an easy consequence.

We state here the essential steps in the construction of Nagata's Example 2. Let $K$ be a field, let $x$ be an indeterminate over $K$, and for $1 \leqslant i \leqslant r$ let $z_{i}=\sum_{j=0}^{\infty} a_{i j} x^{j}$ be elements in $K[[x]]$ that are algebraically independent over $K(x)$. Set $\left(z_{i}\right)_{0}=x z_{i}$ for each $i$, and for $j>0$ set

$$
\left(z_{i}\right)_{j}=\left(z_{i}-\sum_{k<j} a_{k} x^{k}\right) / x^{j-1} \text {. }
$$

We denote by $Z_{i}$ the set $\left\{\left(z_{i}\right)_{j}\right\}_{j=0}^{\infty}$. In the ring $R_{1}=K\left[x,\left\{Z_{i}\right\}_{i=1}^{r}\right]$ the maximal ideals $M=(x)$ and $N=\left(x-1, z_{1}, \ldots, z_{r}\right)$ have rank 1 and rank $r$ +1 , respectively. The basic notion of the construction is to now extend $M$ and $N$ to prime ideals $M^{e}$ and $N^{e}$, respectively, in an appropriate ring $T$ with maximal ideals $M^{\prime \prime}$ and $N^{\prime \prime}$ such that $M^{e} \subset M^{\prime \prime}, N^{e} \subset N^{\prime \prime}$, rank $M^{\prime \prime}=2$, and rank $N^{\prime \prime}=r+2$. Finally, the ideals $M^{\prime \prime}$ and $N^{\prime \prime}$ are "joined" as indicated in the above proposition to form a maximal ideal $P$ in a local ring with saturated chains of prime ideals as follows:

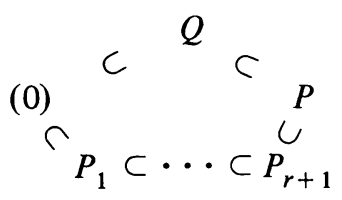

Specifically, let $y$ be an indeterminate over $R_{1}$ and in $R_{2}=R_{1}[y]$ let $M^{\prime}=$ $(x, y)$ and $N^{\prime}=\left(x-1, z_{1}, \ldots, z_{r}, y\right)$. Then $M^{\prime}$ and $N^{\prime}$ are maximal ideals of rank 2 and rank $r+2$, respectively. If $S$ denotes the multiplicative system $R_{2} \backslash\left(M^{\prime} \cup N^{\prime}\right)$, then $\left(\left(R_{2}\right)_{S}, M_{S}^{\prime}, N_{S}^{\prime}\right)$ is a semilocal domain with $\left(R_{2}\right)_{S} / M_{S}^{\prime}$ $\cong\left(R_{2}\right)_{S} / N_{S}^{\prime} \cong K$. By the Proposition $R=K+\left(M^{\prime} \cap N^{\prime}\right)_{S}$ is a local (Noetherian) ring in which $\left(M^{\prime} \cap N^{\prime}\right)_{S} \supset x\left(R_{2}\right)_{S} \cap R \supset(0)$ is a saturated chain and $\left(M^{\prime} \cap N^{\prime}\right)_{S}$ has rank $r+2$. Thus, $x\left(R_{2}\right)_{S} \cap R \subset\left(M^{\prime} \cap N^{\prime}\right)_{S}$ is abnormal of degree $r$.

It is evident that a ring in which the set of degrees of abnormality is unbounded has infinite Krull dimension. Nagata's Example 1 [4, p. 203] is the standard example of a Noetherian ring with infinite Krull dimension and the reader will observe that in our Example 1 we employ some of the techniques used by Nagata in his example.

EXAMPLE 1. A Noetherian ring in which the set of degrees of abnormality is unbounded.

Let $K$ denote a field and let $\left\{x_{i}\right\}_{i=1}^{\infty}$ and $\left\{y_{i}\right\}_{i=1}^{\infty}$ be indeterminates over $K$. 
For each $i$, let $z_{i 1}, \ldots, z_{i i}$ be elements in $K\left[\left[x_{i}\right]\right]$ that are algebraically independent over $K\left(\left\{x_{i}\right\}_{i=1}^{\infty}\right)$ and set $K_{i}=K\left(\left\{x_{j}, Z_{j 1}, \ldots, Z_{j j}, y_{j}\right\}_{j \neq i}\right)$ where as in the preliminary remarks $Z_{j k}=\left\{\left(z_{j k}\right)_{t}\right\}_{t=0}^{\infty}$. If we perform Nagata's construction using

$$
D_{i}=K_{i}\left[x_{i}, Z_{i 1}, \ldots, Z_{i i}, y_{i}\right]
$$

( $D_{i}$ plays the role of the $R_{2}$ in the preliminary discussion), yielding

$$
R_{i}=K_{i}+\left(M_{i} \cap N_{i}\right)_{S_{i}}
$$

where $M_{i}=\left(x_{i}, y_{i}\right) D_{i}, N_{i}=\left(x_{i}-1, z_{i 1}, \ldots, z_{i i}, y_{i}\right) D_{i}$ and $S_{i}=D_{i} \backslash\left(M_{i} \cup\right.$ $\left.N_{i}\right)$, then, as observed above, $R_{i}$ is a local ring and $\left[\left(x_{i} D_{i}\right)_{S_{i}} \cap R_{i}\right] \subset\left(M_{i} \cap\right.$ $\left.N_{i}\right)_{S_{i}}$ has degree of abnormality $i$.

THEOREM. The ring $R=\bigcap_{i=1}^{\infty} R_{i}$ is a Noetherian ring in which the set of degrees of abnormality is unbounded.

Proof. Our approach is to construct a ring $T$ such that each $R_{i}$ is a localization at some prime ideal of $T$. Thus, let $T$ denote the ring $K\left[\left\{L_{i}\right\}_{i=1}^{\infty}\right]$ where $L_{i}=\left(M_{i} \cap N_{i}\right) \cap K\left[x_{i}, Z_{i 1}, \ldots, Z_{i i}, y_{i}\right]$. Since $T / L_{i} T \cong K\left[\left\{L_{j}\right\}_{j \neq i}\right]$, $L_{i} T$ is a prime ideal and so $W_{i}=T \backslash L_{i} T$ is a multiplicative system. We will show that $T_{W_{i}}=R_{i}=K_{i}+\left(M_{i} \cap N_{i}\right)_{S_{i}}$. Let $a / b \in K_{i}$ where $a, b \in$ $K\left[\left\{x_{j}, Z_{j 1}, \ldots, Z_{j j}, y_{j}\right\}_{j \neq i}\right] \backslash(0)$. For each $j$, if $f \in K\left[x_{j}, Z_{j i}, \ldots, Z_{j j}, y_{j}\right] \backslash(0)$, then $y_{j} f \in L_{j} \backslash(0) \subseteq W_{i}$ for $i \neq j$. Thus, if $k_{1}, \ldots, k_{s}$ is a complete list of all the subscripts appearing in $a$ or $b$, then

$$
a / b=\left(a \prod_{t=1}^{s} y_{k_{t}}\right) /\left(b \prod_{t=1}^{s} y_{k_{t}}\right) \in T_{W_{i}}
$$

Therefore $K_{i} \subseteq T_{W_{i}}$. Since $M_{i}$ and $N_{i}$ are comaximal in $D_{i}, M_{i} \cap N_{i}=M_{i} N_{i}$. Since each of $M_{i}$ and $N_{i}$ is generated by elements of $K\left[x_{i}, Z_{i 1}, \ldots, Z_{i i}, y_{i}\right]$, it follows that $L_{i} D_{i}=M_{i} \cap N_{i}$. In fact, $L_{i}$ generates $M_{i} \cap N_{i}$ as a vector space over $K_{i}$. Suppose that $P$ is a prime ideal of $D_{i}$ that contains no elements from the saturation of $W_{i}$ in $D_{i}$, and that $P \& M_{i} \cup N_{i}$. Since $M_{i}$ and $N_{i}$ are the only maximal ideals of $D_{i}$ which contain $M_{i} \cap N_{i}$ and neither contains $P$, it follows that $P$ and $M_{i} \cap N_{i}$ are comaximal. If $p \in P$ and $m \in M_{i} \cap N_{i}$ are such that $p+m=1$, then $p=1-m \in T_{W_{i}} \backslash\left(L_{i} T\right)_{W_{i}}$ and so there exists $d \in W_{i}$ such that $d p \in T \backslash L_{i} T=W_{i}$. Since this contradicts our choice of $P$, we must have $P \subseteq M_{i} \cup N_{i}$ so $S_{i}=D_{i} \backslash\left(M_{i} \cup N_{i}\right)$ is the saturation of $W_{i}$ in $D_{i}$.

We now have $K_{i} \subseteq T_{W_{i}}$ and $\left(M_{i} \cap N_{i}\right)_{S_{i}}=\left(L_{i} T\right)_{W_{i}}$, so it follows that $T \subseteq R_{i} \subseteq T_{W_{i}}$. But $R_{i}$ is a local ring whose maximal ideal is centered on $L_{i} T$ in $T$. Therefore, $R_{i}=T_{W_{i}}$.

Now let $W=\bigcap_{i=1}^{\infty} W_{i}=T \backslash \cup_{i=1}^{\infty} L_{i} T$. We wish to show that $R=$ $\cap_{i=1}^{\infty} R_{i}=T_{W}$. We begin by showing that if $Q$ is a prime ideal of $T$ such that $Q \cap W=\varnothing$-that is, $Q \subseteq \cup_{i=1}^{\infty} L_{i} T$-then $Q \subseteq L_{i} T$ for some $i$. It will then follow from [1, Lemma 5.4] that $R=T_{W}$. Thus, suppose that $Q \subseteq \cup_{i=1}^{\infty} L_{i} T$, 
$Q \notin L_{i} T$ for all $i$, and let $a \in Q, a \neq 0$. Since $a$ involves only finitely many variables it is contained in only finitely many of the ideals $L_{j} T$, say $a \in L_{j} T$ for $1 \leqslant j \leqslant k$ and $a \notin L_{j} T$ for $j>k$. If $Q \subseteq \cup_{j=1}^{k}\left(L_{j} T\right)$, then $Q \subseteq L_{j} T$ for some $j, 1 \leqslant j \leqslant k$, contrary to our assumption. Therefore, there exists $b \in Q \backslash \cup_{j=1}^{k} L_{j} T$ and $(a, b) T \& L_{i} T$ for all $i$. Let $n$ and $m$ be positive integers with $m>n$. No $L_{i} T$ contains both $a+b^{n}$ and $a+b^{m}$, for suppose $a+b^{n}, a+b^{m} \in L_{i} T$. In this case neither $a$ nor $b$ can be in $L_{i} T$ since otherwise they both would be, a contradiction of the choice of $a$ and $b$. Consequently since

$$
\left(a+b^{n}\right)-\left(a+b^{m}\right)=b^{n}-b^{m}=b^{n}\left(1-b^{m-n}\right) \in L_{i} T
$$

and $b \notin L_{i} T$ it follows that $1-b^{m-n} \in L_{i} T$. Since $b \in Q \subseteq \bigcup_{j=1}^{\infty} L_{j} T, b$ has zero constant term and $1-b^{m-n}$ has constant term 1 . But no such element can be contained in $\cup_{j=1}^{\infty} L_{j} T$ 's. Hence $a+b^{n}$ and $a+b^{m}$ are in distinct $L_{i} T$ 's. This shows that the set $\left\{a+b^{n}\right\}_{n=1}^{\infty} \subseteq Q \subseteq \cup_{j=1}^{\infty} L_{j} T$ cannot be contained in any finite union of the $L_{j} T$. But, to the contrary, since $a$ and $b$ each involve only a finite number of indeterminates, $\left\{a+b^{n}\right\}_{n=1}^{\infty}$ is contained in only finitely many $L_{j} T$ 's. We now have the desired conclusion that $Q \subseteq L_{i} T$ for some $i$. Hence

$$
T_{W}=\bigcap_{i=1}^{\infty} T_{L T}=\bigcap_{i=1}^{\infty} T_{W_{i}}=\bigcap_{i=1}^{\infty} R_{i}=R .
$$

Since $R_{i}=T_{W_{i}}=\left(T_{W}\right)_{(L, T)_{W}}=R_{\left(L_{i} T\right)_{w}}, R$ is locally Noetherian and has an abnormality of degree $i$ for each positive integer $i$. If $f \in R, f \neq 0$, then as observed above, $f$ is contained in only a finite number of maximal ideals. By [4, E1.1, p. 203] $R$ is a Noetherian ring and the proof is complete.

EXAMPLE 2. A local domain with prime ideals $I_{1} \subset I_{2}$ that are abnormal of degree 2 but such that no minimal element of $\left\{I \mid I\right.$ is a prime ideal and $I_{1} \subset I$ is abnormal $\}$ is 2-abnormal over $I_{1}$.

Let $K$ be a field of characteristic 2 with $|K|>2$ and let $x, w, z \in K[[x]]$ be algebraically independent over $K$. We write

$$
w=\sum_{i=0}^{\infty} a_{i} x^{i} \text { and } z=\sum_{i=0}^{\infty} b_{i}[x(x+1)]^{i}
$$

and define $w_{0}=x w, w_{i}=\left(w_{i-1} / x\right)-a_{i-1}$ for $i>0$. Similarly, we define

$$
z_{0}=[x(x+1)] z \text { and } z_{i}=\left[z_{i-1} / x(x+1)\right]-b_{i-1}
$$

for $i>0$. If $R_{1}=K\left[x,\left\{w_{i}\right\}_{i=0}^{\infty},\left\{z_{i}\right\}_{i=0}^{\infty}\right]$ then $M_{1}=(x), M_{2}=(x+1, w)$, and $M_{3}=(x+\beta, w, z), \beta \neq 0,1$, are maximal ideals of $R_{1}$ such that $R_{1} / M_{i}=K$ for $i=1,2,3$. Note that $\left(R_{1}\right)_{(x)}$ is a discrete rank one valuation ring (DVR) (since $\left(R_{1}\right)_{(x)}$ is dominated by $K[[x]]-$ cf. $[4$, p. 204]).

Claim. $\left(R_{1}\right)_{M_{2}}$ is a two dimensional Noetherian ring.

For the same reason that $\left(R_{1}\right)_{(x)}$ is a DVR, $K\left[x,\left\{z_{i}\right\}_{i=0}^{\infty}\right]_{(x)}$ is a DVR. Consider the automorphism $\sigma$ on $K(x, z)$ such that $\sigma(x)=x+1$ and $\sigma(z)=$ $z$. Since $\sigma(x(x+1))=x(x+1), \sigma\left(z_{i}\right)=z_{i}$ for all $i$ and $\sigma$ determines an 
automorphism on $K\left[x,\left\{z_{i}\right\}\right]$. It follows that $K\left[x,\left\{z_{i}\right\}\right]_{(x+1)} \cong K\left[x,\left\{z_{i}\right\}\right]_{(x)}$ and so $K\left[x,\left\{z_{i}\right\}\right]_{(x+1)}$ is a DVR. Hence

$$
K\left[x,\left\{z_{i}\right\}, w\right]_{(x+1, w)}=\left(K\left[x,\left\{z_{i}\right\}\right]_{(x+1)}\right)[w]_{(x+1, w)}
$$

is a two dimensional local ring. Since $1 / x$, and hence $w_{i}$ for each $i$, is in $K\left[x,\left\{z_{i}\right\}, w\right]_{(x+1, w)}$, we have $\left(R_{1}\right)_{(x+1, w)}=K\left[x,\left\{z_{i}\right\}, w\right]_{(x+1, w)}$ and the claim is established.

Since $R_{1}[1 / x, 1 /(x+1)]=K[x, 1 / x, w, 1 /(x+1), z]$, the ideal $M_{3} R_{1}[1 / x, 1 /(x+1)]$ is a rank 3 prime and so $M_{3}$ is a rank 3 prime ideal in $R_{1}$. We have thus shown that for each $i=1,2,3$, rank $M_{i}=i$ and $\left(R_{1}\right)_{M_{i}}$ is Noetherian. We will now use essentially Nagata's construction as previously outlined to "join" maximal ideals containing appropriate extensions of $M_{1}$ and $M_{2}$. Let $y$ be an indeterminate over $R_{1}$ and in $R_{2}=R_{1}[y]$ consider the ideals $A=(x, y), B=(x+1, w, y)$ and $C=(x+\beta, w, z, y)$. If $T=$ $R_{2} \backslash(A \cup B)$ then, following Nagata's construction, $R=K+(A \cap B)_{T}$ is a local ring with maximal ideal $P_{1}=(A \cap B)_{T}$ of rank 3, the prime ideal $Q_{1}=x\left(R_{2}\right)_{T} \cap R$ has rank one, and $P_{1} \supset Q_{1} \supset(0)$ is a saturated chain. Therefore $Q_{1} \subset P_{1}$ is 1-abnormal.

We now wish to show that the ring $S_{1}=R \cap\left(R_{2}\right)_{C}$ is a semilocal ring whose only maximal ideals are $P_{2}=P_{1} \cap S_{1}$ of rank 3 and $G_{1}=C\left(R_{2}\right)_{C} \cap$ $S_{1}$ of rank 4 and that $R=\left(S_{1}\right)_{P_{2}}$ and $\left(R_{2}\right)_{C}=\left(S_{1}\right)_{G_{1}}$. To do this we first prove that both $R$ and $\left(R_{2}\right)_{C}$ are localizations of $K[A \cap B]$. First, we show that $R=(K[A \cap B])_{T_{1}}$ where $T_{1}=K[A \cap B] \backslash(A \cap B)$. Clearly, since $T_{1} \subseteq T$,

$$
R \supseteq(K[A \cap B])_{T_{1}} \supseteq K+(A \cap B)_{T_{1}},
$$

so it suffices to show that $(A \cap B)_{T_{1}}=(A \cap B)_{T}$. Considering $T_{1}$ as a multiplicative system in $R_{2}$, let $P$ be a prime ideal of $R_{2}$ such that $P \cap T_{1}=$ $\varnothing$. If $P \& A \cup B$ then, since $A$ and $B$ are maximal ideals in $R_{2}, P$ is comaximal with $A \cap B$. Hence there exists elements $p \in P$ and $a \in A \cap B$ such that $p+a=1$. But then $p=1-a \in P \cap T_{1}$ contrary to the choice of $P$. It follows that $P \subseteq A \cup B$ and so $T=R_{2} \backslash(A \cup B)$ is the saturation of $T_{1}$ in $R_{2}$. Consequently,

$$
(A \cap B)_{T_{1}}=(A \cap B)_{T} \quad \text { and } \quad R=(K[A \cap B])_{T_{1}}
$$

Next we show that $\left(R_{2}\right)_{C}=(K[A \cap B])_{C \cap K[A \cap B]}$. To see that

$$
\left(R_{2}\right)_{C} \subseteq(K[A \cap B])_{C \cap K[A \cap B]}
$$

let $a / b \in\left(R_{2}\right)_{C}$, where $a \in R_{2}, b \in R_{2} \backslash C$. Then

$$
a / b=[a x(x+1)] /[b x(x+1)] \in(K[A \cap B])_{C \cap K[A \cap B]^{\circ}}
$$

Since the opposite containment is obvious, we have established the equality.

It now follows that

$$
\begin{aligned}
S_{1} & =R \cap\left(R_{2}\right)_{C}=(K[A \cap B])_{A \cap B} \cap(K[A \cap B])_{C \cap K[A \cap B]} \\
& =(K[A \cap B])_{(K[A \cap B]) \backslash((A \cap B) \cup(C \cap K[A \cap B]))}
\end{aligned}
$$


so $S_{1}$ is a semilocal ring with maximal ideals $P_{2}=P_{1} \cap S_{1}$ and $G_{1}=C\left(R_{2}\right)_{C}$ $\cap S_{1}$. Further, $\left(S_{1}\right)_{P_{2}}=R$ and $\left(S_{1}\right)_{G_{1}}=\left(R_{2}\right)_{C}$, so rank $P_{2}=3$ while rank $G_{1}$ =4. Recall that in $R, Q_{1}=x\left(R_{2}\right)_{T} \cap R$ is a rank one prime ideal such that $P_{1} \supset Q_{1} \supset(0)$ is a saturated chain. If we define $Q_{2}=Q_{1} \cap S_{1}$, then $Q_{2} \underline{Z} G_{1}$ since $x(x+1) \in Q_{2} \backslash G_{1}$. Therefore, $P_{2}$ is the only prime ideal of $S_{1}$ which properly contains $Q_{2}$. It follows that in $S_{1}$ we have satuated chains of prime ideals as follows:

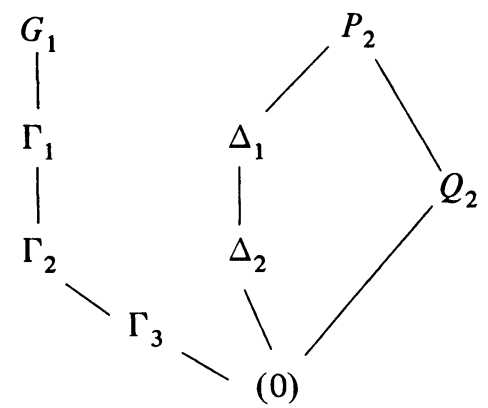

Now let $S_{2}=S_{1}[u], E=\left(P_{2}, u\right)$, and $G=\left(G_{1}, u\right)$ where $u$ is an indeterminate over $S_{1}$. Since $P_{2}$ is minimal over $Q_{2}$ in $S_{1}, P_{2}[u]$ is a rank 3 prime ideal in $S_{2}$ that is minimal over $Q_{2}[u]$ and $\operatorname{rank}\left(E / Q_{2}[u]\right)=2$. If $V$ is any prime ideal of $S_{2}$ that is minimal over $Q_{2}[u]$ then, since $P_{2}$ is the only prime ideal of $S_{1}$ properly containing $Q_{2}$, either $V=P_{2}[u]$ or $V \cap S_{1}=Q_{2}$. Hence, if $V \neq$ $P_{2}[u]$ then rank $V=\operatorname{rank} Q_{2}+1=2\left[3\right.$, Theorem 39]. Therefore $P_{2}[u]$ is the only prime ideal of $S_{2}$ with rank more than 2 that is minimal over $Q_{2}[u]$. Note also that since $Q_{2} \& G_{1}, Q_{2}[u] \& G=\left(G_{1}, u\right)$.

We now perform Nagata's construction (with $S_{2}$ playing the role of the $R_{2}$ of the preliminary discussion) and "join" appropriate extensions of $E$ and $G$. Specifically if we set $S=K+(E \cap G)_{T_{3}}$, where $T_{3}=S_{2} \backslash(E \cup G)$, then by the proposition in the preliminary remarks, $S$ is the desired ring where $I_{1}=\left(Q_{2}[u]\right)_{T_{3}} \cap S$ and $I_{2}=(E \cap G)_{T_{3}}$.

\section{REFERENCES}

1. R. Gilmer, Multiplicative ideal theory, Marcel Dekker, New York, 1972.

2. E. G. Houston and S. McAdam, Rank in Noetherian rings, J. Algebra 37 (1975), 64-73.

3. I. Kaplansky, Commutative rings, Allyn and Bacon, Boston, Mass., 1970.

4. M. Nagata, Local rings, Interscience, New York, 1962.

5. L. J. Ratliff, Jr., Characterizations of catenary rings, Amer. J. Math. 93 (1971), 1070-1108.

Department of Mathematics, Virginia Polytechnic Institute and State University, Blacksburg, Virginia 24061 